\title{
Springer
}

Draft Manuscript for Review

\section{Effects of different cooking conditions on the anthocyanin content of a black rice (Oryza sativa L. 'Violet Nori')}

\begin{tabular}{|r|l|}
\hline Journal: & European Food Research and Technology \\
\hline Manuscript ID & EFRT-19-0003.R1 \\
\hline Danuscript Type: & Original paper \\
\hline Author: & n/a \\
\hline & $\begin{array}{l}\text { Catena, Silvia ; University of Genoa, Department of Pharmacy } \\
\text { Turrrini, Federica; University of Genoa, Department of Pharmacy } \\
\text { Boggia, Raffaella; University of Genoa, Department of Pharmacy } \\
\text { Borriello, Matilde; Marco Polo Hotel School } \\
\text { Gardella, Marco; Marco Polo Hotel School } \\
\text { Zunin, Paola; University of Genoa, Department of Pharmacy }\end{array}$ \\
\hline Keywords: & $\begin{array}{l}\text { 'Violet Nori' rice (<i>Oryza sativa</i> L.), anthocyanins, cooking tests, } \\
\text { antioxidants }\end{array}$ \\
\hline
\end{tabular}

\section{SCHOLARONE ${ }^{m}$ Manuscripts}




\section{Effects of different cooking conditions on the antioxidant anthocyanin content of a violet black rice (Oryza Sativa sativa $L$. 'Violet Nori')}

Silvia Catena ${ }^{1}$, Federica Turrini ${ }^{1}$, Raffaella Boggia ${ }^{1}$, Matilde Borriello ${ }^{2}$, Marco Gardella ${ }^{2}$ and Paola Zunin ${ }^{1 *}$

${ }^{1}$ Department of Pharmacy, University of Genova, Viale Cembrano 4, 16148, Genova, Italy

${ }^{2}$ Marco Polo Hotel School, Via Angelo Sciaccaluga 9, 16147, Genova, Italy.

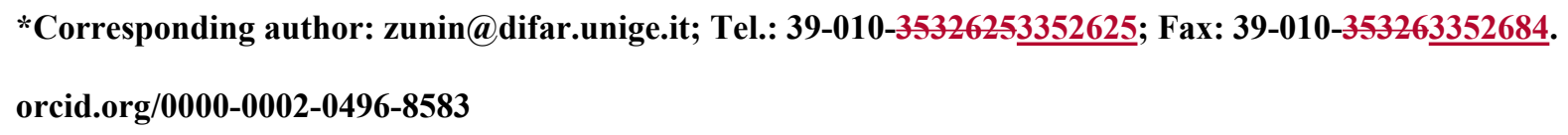

Acknowledgement: The authors are grateful to Azienda Agricola Eleonora Bertolone for the rice supplying. 
The study is focused on the effect of different cooking conditions on the antioxidant content, particularly anthocyanins, of Oryza s Sativa L. 'Violet Nori, a new violet black rice cultivar. 10 different cooking tests were performed. The selected cooking conditions allowed to evaluate the effect of boiling, roasting, oven cooking, risotto cooking, and oriental cooking. The total anthocyanins amounts were evaluated by both a spectrophotometric pH differential method and HighPerformance Liquid Chromatography (HPLC), together with the Total Phenolic Content (TPC) and the Radical Scavenging Activity (RSA). The obtained results showed that boiling with a low water amount (100 g rice/650 mL water) and oriental cooking, which allows a reduction of boiling times thanks to the lid of the pota sealing cover, allows effectively saving at least part of their anthocyanins content. Similar results were obtained by boiling if rice was introduced in cold or boiling water, but on the contrary a ratio of $100 \mathrm{~g}$ rice-/1000 $\mathrm{mL}$ water greatly enhanced the loss of antioxidant compounds. Risotto and oven cooking allowed obtaining results that were roughly intermediate between the 'best' and the 'worst' results. A high correlation existed between the amounts of total anthocyanins and both TPCs and RSAs. The 'best' results show that although cooking necessarily decreases the rice content of valuable antioxidants, a careful choice of the operative conditions allows effectively preserving amounts of total anthocyanins higher than 100

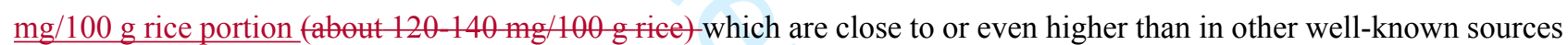
of dietetic anthocyanins. 
Keywords: ‘Violet Nori’ rice (Oryza șSativa L.), anthocyanins, cooking tests, antioxidants.

\section{Introduction}

The interest in the healthy properties of eolourpigmented rice is continuously expanding all over the world and in Italy its crop areas have increased more than seven times in the last years [1]. Several bioactive compounds have been identified in the coloured caryopses, most belonging to the class of phenolic compounds [2-5]. Anthocyanins, the major water-soluble pigments which accumulate in the grains during maturation $[4,6]$, are principally known for their antioxidant activity [7-10]. Moreover in 2016 an exhaustive review by Olivas Aguirre et al. [11] dealing with cyanidin3-O-glucoside, which is by far the major anthocyanin in rice, underlines its intense anti-proliferative effect against different kinds of cancer cell, even though the amounts used both for in vitro tests and in laboratory animals were often higher than those obtainable from food sources. As far as the anti-inflammatory effect of anthocyanins is concerned, in 2015 Vendrame and Klimis-Zacas [12] examined the scientific literature on this matter: they concluded that, on the basis of both in vivo and in vitro evidences, these properties are to be mainly attributed to the anti-oxidant properties of anthocyanins, though other mechanisms could be partially involved and still need to be clarified. More generally, Olivas Aguirre reported that several biological activities of dietary anthocyanins, such as their cancer preventive activity [13], their anti-inflammatory one $[12,14]$ and their possible role in the prevention of cardiovascular diseases (CVD) [15] are probably related to their primary antioxidant activity [11].

Recently, Zhu [16] confirmed that anthocyanins have both in vitro and in vivo antioxidant and retinal protection activities, together with the ability to inhibit cholesterol adsorption and to regulate lipid profile. Other interesting biological activities of dietary anthocyanins are their glycaemic regulation activity, at present confirmed only by in vitro tests [16], and their neuroprotective effect, probably due to the modulation of gut microbiota $[16,17,18]$.

In addition, other classes of flavonoids having similar protective activity, such as flavonols and flavan-3-ols, have been identified in eolourpigmented rice, and the positive interaction of anthocyanins with them is now clearly established [16]. However, it is interesting to underline that after oral introduction anthocyanins have a peculiar metabolic fate, since they can be partially absorbed in their native form in the stomach [19,20], after release from the food matrix thanks to the acidic conditions $[16,21]$. However, many studies report that in the human body anthocyanins reach the highest concentration in intestine [18], thus the gastric adsorption is not relevant to the effect of anthocyanins on the axis gut-brain. Although some authors reported that only the $9-10 \%$ of the intact anthocyanins can be absorbed in the stomach [11], a correct evaluation of the bio-activity of dietary anthocyanins should consider the concentrations of both the parent molecules and of their active metabolites [18]. Moreover, they are also absorbed in their intact form in the intestinal wall, where they can reach significant concentrations before undergoing a transformation to phenolic acids, thanks to the activity of colonic bacterial metabolism [18]. These acids, particularly the major metabolite protocatechuic 
acid, are highly concentrated in the blood stream and probably responsible of another part of the biological activity of anthocyanins $[18,19]$.

Despite the great interest in the anthocyanins metabolism and bio-activity, few studies have been published about the effect of cooking on the content of anthocyanins and phenolic compounds in colourpigmented rice [22, 24] and in 2009 Hiemori et al. reported a dramatic decrease in the anthocyanins content after cooking [23]. Thus, the aim of this study was to investigate if the residual amounts of these compounds after cooking could yet support the inclusion of colourpigmented rice among the most interesting sources of dietary anthocyanins and antioxidant compounds. The study is particularly focused on Oryza Sativa sativa L. 'Violet Nori', a new variety of violet black rice growing in Piedmont and registered at the Community Plant variety Office, whose anthocyanins content is generally higher or at least comparable with that in other best known black cultivar, such as 'Venere', 'Nerone', and 'Artemide'[1].

\section{Materials and Methods}

\section{Chemicals}

Cyanidine-3-O-glucoside chloride, DPPH• (1,1-diphenyl-2-picrylhydrazyl), Folin-Ciocalteu reagents, gallic acid, Trolox (6-hydroxy-2,5,7,8-tertramethylchromane-2 carboxylic acid), ethyl alcohol p.a., acetonitrile HPLC grade and formic acid were supplied by SIGMA. Deionised-Deionized water $(18 \mathrm{M} \Omega)$ was produced by a Milli-Q system (Millipore, USA).

\section{Equipment.}

A Retsch Grindomix 200 M mill (Haan, Germany) and an IKA Ultra-Turrax T25 (Staufen im Breisgau, Germany) were employed for the homogenization of the samples in the extraction solvents. The direct Ultrasound Assisted Extraction was realized by Hielscher UP200St (Teltow, Germany). An UV-Vis Agilent 8453 (Waldbronn, Germany) allowed the determinations of the Total Phenolic Content (TPC) [25], Radical Scavenging Activity (RSA) and Total Anthocyanins by the $\mathrm{pH}$ differential method [26] in the extracted solutions. An Agilent 1100 Liquid chromatograph equipped with a Diode Array Detector (DAD) allowed the HPLC determination of anthocyanins [1].

5-Samples: 'Violet Nori’ whole rice was supplied by Azienda Agricola Eleonora Bertolone (Collobiano, VC, Italy), which collected it in 2017.

\section{Cooking treatments}

The cooking tests were carried out employing the same rice batch. Ten different cooking tests were performed with professional cooking equipment at the Hotel School, Genova. For each test $100 \mathrm{~g}$ of whole 'Violet Nori' rice were cooked either in pan or oven by employing different conditions, which are summarized in Table 1. 
Boiling (tests 1-4). $100 \mathrm{~g}$ dried rice were added to 650 or $1000 \mathrm{~mL}$ of cold $\left(20^{\circ} \mathrm{C}\right)$ or boiling $\left(100{ }^{\circ} \mathrm{C}\right)$ water; cooking required $40 \mathrm{~min}$ after return to boiling.

'Oriental' cooking (test 5). $100 \mathrm{~g}$ dried rice were added to $300 \mathrm{~mL}$ of boiling water $\left(100^{\circ} \mathrm{C}\right)$, boiled without covering for $10 \mathrm{~min}$ and then, with a tight sealed coverlid on the pot, for $14 \mathrm{~min}$, up to the incorporation of the cooking water.

'Risotto' cooking (tests 6 and 7). Although this peculiar Italian cooking style normally involves a slow cooking, under a careful mixing, covering the rice grains with hot broth, a significant variation was adopted in order to reduce the cooking times of the whole rice and to better preserve the water-soluble compounds. Thus, a preliminary 10 min boiling of $100 \mathrm{~g}$ dried rice in boiling water was realized. The boiling water was then drained and collected, and a classical 'risotto' cooking was realized by employing the discarded and intensely coloured boiling water instead of broth, with or without a preliminary rice toasting. The amounts of the initial water for the test with and without the preliminary roasting were $750 \mathrm{~mL}$ and $1000 \mathrm{~mL}$, respectively.

Oven cooking (tests 8-10). Rice was prepared in a kitchen oven employing an uncovered baking tray. The effects of toasting (test 9) and of a preliminary 10 min boiling (test 10) were also tested.

In order to evaluate the initial anthocyanin content, a control test (test 11) was prepared by drying, grinding and extracting the crude whole violet rice in the same conditions of the cooked samples.

\section{Extraction of anthocyanins and other antioxidant compounds}

After cooking at the Hotel $\underline{S} s c h o o l$ Marco Polo (Genova), the samples were immediately transferred to the University laboratories and oven dried at $40{ }^{\circ} \mathrm{C}$ for 48 hours. A Grindomix $200 \mathrm{M}$ mill (Retsch) (35 sec at 5000 rpm) finely ground the grains: $1 \mathrm{~g}$ powder was then suspended in $40 \mathrm{~mL}$ EtOH$/ \mathrm{H}_{2} \mathrm{O}$ mixture $(60: 40 \mathrm{v} / \mathrm{v})$ and homogenized by an UltraTurrax T25 homogenizer (IKA) in an ice bath $(0.5 \mathrm{~min}$ at $8000 \mathrm{rpm}$, followed by $1.5 \mathrm{~min}$ at $24000 \mathrm{rpm})$. The final extraction was obtained by the direct ultrasound assisted extraction method optimized and described in a previous study [1]. The extracted solutions were separated by centrifugation (3000 rpm for $10 \mathrm{~min}$ ), followed by a Whatmann $\mathrm{n} .1$ paper disk filtration of the supernatant for the separation of the coarse solid material and a further filtration by an $\mathrm{RC}$ membrane syringe filter $(0.45 \mu \mathrm{m})$ for the removal of the fine solid material. Two replicated extractions were performed for each sample.

\section{Analysis of the extracted solutions}

The total anthocyanins content was determined both by the previously described HPCLHPLC-DAD method [1]_(Figure 1) and by the spectrophotometric $\mathrm{pH}$ differential method [27]. The results were expressed as $\underline{\text { as } \mu \mathrm{g} \text { cyanidin-3- }}$ 
glucoside $/ \mathrm{mL}$ in the extracted solutions and turned into $\mathrm{mg} / 100 \mathrm{~g}$ crude rice on a dried matter basis (d.m.), using cyanidin-3-glucoside in the range between 1 and $100 \mu \mathrm{g} / \mathrm{mL}$ as external standard.

Other two tests were performed by UV-VIS spectrometry: the DPPH test [26] for the determination of the RSA, expressing the results as $\mu \mathrm{mol}$ TEAC/g sample en a dried matter basis (d.m.), and the Folin-Ciocalteau test [25] which determines the value of the TPC, expressing the results as mg GAE/g sample d.m..

As far as DPPH test is concerned, briefly a DPPH radical solution approximately $10^{-4} \mathrm{M}$ in methanol was daily prepared. The initial DPPH concentration in this solution was measured by the absorbance at $515 \mathrm{~nm}$ of the control sample $\mathrm{C}_{0}$ (without extracts), which was obtained by diluting $0.250 \mathrm{~mL}$ of methanol with the DPPH solution into a 10 $\underline{\mathrm{mL} \text { volumetric flask. Then, in order to measure the antiradical activity of each extract, } 0.250 \mathrm{~mL} \text { of the extracted }}$ solution were transferred into a $10 \mathrm{~mL}$ volumetric flask before adding the DPPH solution to the mark. The flask was gently shaken and kept in the dark for 30 min before reading the residual absorbance. Before each sample a blank absorbance $(0.250 \mathrm{~mL}$ of the extracted solution diluted to $10 \mathrm{~mL}$ by pure methanol, without $\mathrm{DPPH})$ was measured and then subtracted. For each sample, the amount of the reacted DPPH was obtained by subtracting the residual absorbance at $515 \mathrm{~nm}$ from the $\mathrm{C}_{0}$ absorbance, and was transformed in antioxidant activity using 6-hydroxy-2,5,7,8-tetramethyl-2carboxylic acid (Trolox) as reference standard (Trolox Equivalent Antioxidant Capacity, TEAC). Two replicated determinations were performed for each sample.

For the Folin-Ciocalteu test, after a 1:10 dilution of the extracted solution, $0.2 \mathrm{~mL}$ of the diluted solution, $1 \mathrm{~mL}$ of Folin-Ciocalteu reagent (diluted 1:10 with deionized water) and $0.8 \mathrm{~mL}$ of aqueous sodium carbonate $7.5 \% \mathrm{w} / \mathrm{v}$ were mixed together in a test tube and vortexed. After standing in the dark at room temperature $\left(25 \pm 2^{\circ} \mathrm{C}\right)$ for $30 \mathrm{~min}$, the absorbance was read at $760 \mathrm{~nm}$ and the total phenolic concentration was calculated using gallic acid for the calibration curve (Gallic Acid Equivalent, GAE). Two replicated determinations were performed for each sample.

\section{Statistical analysis}

Statistical analysis were performed by the MedCalc ${ }^{\circledR}$ statistic software, freely downloadable for a trial at https://www.medcalc.org/. ANOVA was employed to evaluate the statistical significance of the differences among samples.

\section{Results and diseussion}


As previously reported for other black rice [28], all the HPLC plots showed that cyanidine-3-glucoside was by far the major anthocyanin (about $90 \%$ of total anthocyanins) in 'Violet Nori' caryopses, both before and after cooking, whereas peonidin-3-glucoside stood for about the $8-10 \%$ of total anthocyanins. Only minor amounts of 2 other anthocyanins were detected (Figure1). The ratios among anthocyanins were almost preserved in the cooking tests, though in some samples (i.e. tests 3 and 4) only cyanidin-3-glucoside and peonidin-3-glucoside were detectable after cooking. Considering its simplicity, its low cost and low environmental impact, the pH differential method for the total anthocyanins determination [277] was thus tentatively applied together with the previously employed HPLC-DAD method with the aim of comparing the results obtained by the two methods, both employing cyanidine-3-glucoside as standard reference. In order to evaluate if the two methods give comparable results, the obtained values of the total anthocyanins amount in the extracts obtained after the ten cooking tests were compared by Passing-Bablok regression (Figure 2), since the classical univariate regression is not fit for the comparison of two variables which are both affected by an experimental error. Although a high correlation existed between the two series of results $\left(\mathrm{R}^{2}=0_{2}-981\right)$, with and there was-no deviation from linearity, the two methods (Figure)-were not statistically ( $p<0,001)$ identical $(p<0,001)$. In fact, the +3.473 intercept $(\neq 0)$ and the 1.134 slope $(\neq 1)$ highlight the probable presence of non-separated coloured compounds, which enhanced the values obtained by the directs spectrophotometric analysis.

As far as the remaining anthocyanins content in the cooked samples is concerned, in the 10 cooking tests (tests 1-10) it $\underline{\text { always appears statistically eertainly lower }(\mathrm{p}<0,001)}$ than in the crude rice (test $11,466 \pm 30 \mathrm{mg} / 100 \mathrm{~g})$ (Figure $z \underline{3})$, with a reduction between $72 \underline{70}$ and $92 \%$. Nevertheless, in some samples, for example those obtained in tests $\underline{1,2}$ and 5 , the residual amounts of anthocyanins were $120-140 \mathrm{mg} / 100 \mathrm{~g}$ rice. The best results $(138 \pm 6 \mathrm{mg} / 100 \mathrm{~g})$ were obtained in the cooking test 5 , which involved the employment of water in a 1:3 (p/v) rice /water ratio, its total adsorption into rice grains within the cooking times and the reduction of the cooking time thanks to the increase of the pressure in the pot due to the sealed covertight lid. The results obtained in the tests 1 and 2 were close to $120 \mathrm{mg} / 100 \mathrm{~g}$ rice, i.e slightly but not significantly $(p<0,05)$ lower than in the test 5 but still satisfying, close to $120 \mathrm{mg} / 100 \mathrm{~g}$ rice: in these tests the rice/water ratio was enhanced at 1:6.5, since the longer cooking time makes it essential to avoid rice drying. Since in these tests too the added water was completely adsorbed, the anthocyanins decrease with respect to test 5 was probably related to the longer boiling time. No statistically significant difference $(\mathrm{p}<0.05)$ - was observed between sample 1 and 2 , i.e. if rice was added to cold water $\left(\mathrm{T}=20^{\circ} \mathrm{C}\right)$ (test 1$)$ or boiling water (test 2$)$. As far as the samples appearance is concerned, the appearance and texture after the 5 test was-were by far more attractive: in fact after oriental cooking rice grains were well-separated, with a bright violet color and a shiny appearance, whereas -the two boiled samples appeared rather sticky. 
$\underline{\text { In the simple and traditional Pilaf cooking (test } 8) \text { anthocyanins were lost in a larger extent than in test } 1 \text { and } 2(p<0.1)}$ although their amount was still close to $100 \mathrm{mg} / 100 \mathrm{~g}$, whereas Risotto (tests 6 and 7 ) and Pilaf + roasting and Pilaf + $\underline{\text { boiling (tests } 8,9 \text { and 10, respectively) and Risotto cooking (tests } 6 \text { and 7) eooking caused much significant reductions }}$

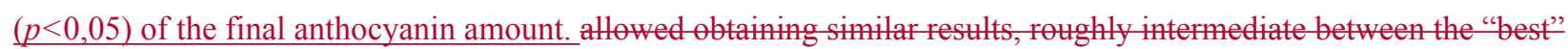
and the "worst" results.As far as risotto is concerned, the preliminary ten minutes boiling and the substitution of broth with the discarded boiling water was not enough to preserve anthocyanins (test 6), and toasting (sample-test 7) led to a sticky consistence. Pilaf oven cooking, both with and without preliminary rice roasting or boiling, did not lead to the hoped results: the final aqueous consistency of rice grains and the amounts of preserved anthocyanins were not satisfactory, although the ratios rice/water were close to those of test 1 and 2 .

As can be expected, as far as anthocyanins amounts are concerned the worst cooking conditions were by far those tested in tests 3 and 4 , which preserved significantly lower amount $(p<0.02)$ than in Risotto and Pilaf samples. It is possible to presume that since the high rice/water ratio $(1: 10)$ had caused a high loss of these compounds in the discarded residual water, and this loss was similar if rice was had been introduced in cold water or at its first boil or in cold water.

With respect to TPCs and RSAs, the detected values appeared highly correlated with the total content of anthocyanins. In order to evaluate this correlation, the crude rice sample was not considered in sight of its possible leverage. When the 10 cooking tests are considered, the correlation between the anthocyanins amounts expressed as $\mathrm{mg} / 100 \mathrm{~g}$ crude rice detected by the HPLC method and the RSAs appeared very high, with $r \underline{\mathrm{R}}=0_{2}=9998$; this value confirms the powerful antiradical activity of the extracted anthocyanins after heating and the possibility to partially preserve this valuable activity even after cooking. The correlation between the anthocyanins content by HPLC and the TPCs appeared slightly lower but still high $\left(\underline{\mathrm{R}} \mathrm{f}=0_{-, 2} 9319\right)$ : the residuals plot shows the higher scatter for test 7 , when rice had undergone the risotto cooking with a 3 min preliminary toasting that probably had determined a thermal degradation of anthocyanins. Similar results were obtained for the correlation between the total anthocyanins detected by the $\mathrm{pH}$ differential method and both TPCs $(\mathrm{R}=0,9574)$ and RSAs $(0,9902)$, all expressed on a dry matter basis (Figure $3 \underline{4})$. Nevertheless, the correlation with RSAs appeared slightly lower than with HPLC-DAD, thus confirming the spectrophotometric interference of molecules which do not belong to the class of anthocyanins and are indeed separated by HPLC.

\section{Discussion}

The comparison between Though the fast UV-VIS and the HPLC methods for the determination of total anthocyanins confirmed that the $\mathrm{pH}$ differential method may slightly overestimate the anthocyanin content [29] since the two $\underline{\text { methods }}$ are not statistically identical, but their high correlation and the high similarity of the obtained results allow to 

rice.

$\underline{\text { As far as the cooking tests are concerned, pressure boiling was not tested since previous study had shown that it causes }}$ the higher losses of anthocyanins $[23,24]$. The cooking tests confirmed that cooking always significantly decreases rice anthocyanins $[23,24]$, thus negatively affecting the RSAs. Nevertheless, tThe results obtained in the cooking tests of 'Violet Nori', show that a careful choice of the cooking conditions allows preserving its healthy peculiar features although cooking necessarily decreases its content of valuable anthocyanins and total phenolic compounds, thus negatively affecting the RSAs. The detected loss of total anthocyanin was in the $70-92 \%$ range, i.e. not far from the previously reported $65,4-74,2 \%$ for cyiandin-3-glucoside [23] and from the average degradation rate of $73,5 \%$ and $\underline{72,5 \% \text { for cyanidin-3-glucoside and peonidin-3 glucoside, respectively [24]. Since neutral water was employed for the }}$ cooking tests, it can be assumed the flavylium cation had been broken in the B ring to form protocatechuic acid [30], which in this study was only tentatively identified but not quantified at $280 \mathrm{~nm}$. The detected loss was also in agreement with the data reported by Zaupa et al. [31] for boiled rice, whereas the better performances that the same authors obtained for risotto were not confirmed. It is possible to assume that the lab-conditions of the risotto cooking [31], i.e. boiling in a test tube up to water sdsorption, had a different effect from the conditions of the 'real' risotto cooking: the direct gas heating of the pan probably involves a higher temperature on the inner pot surface, thus promoting anthocyanin thermal degradation. On the other hand, the conditions applied in lab [31] are much similar to the conditions employed in test 5 , giving the best results. It is also interesting to note that the oriental cooking conditions employed in test 5 are quite similar to the conditions of a rice porridge preparation (without milk) and that porridge preparation has been previously identified as the better cooking method both for waxy and non-waxy black rice [24].

$\underline{\text { As far as the individual anthocyanin is concerned, in accordance with some previous studies }[23,24] \text { no differences }}$ were observed in the tested cooking conditions in the percentage of losses of peonidin-3-glucoside and cyianidin-3glucoside, which were on the contrary highlighted in the study of Zaupa et al. [31] reporting a higher stability of peonidin-3-glucoside.

The reduction of total anthocyanins was always paired with a reduction of TPC and RSA, thus to a reduction of the $\underline{\text { ability for free radical scavenging. Nevertheless, since the amount of anthocyanins in the crude 'Violet Nori' rice was }}$ generally higher than in other reported black rice $[23,24,31,32]$, aAfter 'Violet Nori' cooking, the total amount of anthocyanins ean bewas preserved at values higher than $100 \mathrm{mg} / 100 \mathrm{~g}$ portion when the cooking water is completely adsorbed. The comparison between tests $1,2,3,4$ and 5 shows the importance of the ratio $\mathrm{g}$ rice/mL water in cooking 
operations, since increasing the amounts of the employed water caused a significant loss of antioxidant compounds in the discarded water.

MoreoverOtherwise, the values obtained in the a $100 \mathrm{~g}$ portion in the 'best' cooking conditions are comparable to or even higher than the values reported in the fresh tissues of those fruits and vegetables which are generally considered the best sources of dietetic anthocyanins [ $2 \underline{33} 8]$, such as cranberries $(140 \pm 30 \mathrm{mg} / 100 \mathrm{~g})$, black plums $(140 \pm 26$ $\mathrm{mg} / 100 \mathrm{~g})$, red raspberries $(92 \pm 20 \mathrm{mg} / 100 \mathrm{~g})$, red grapes $(27 \pm 11 \mathrm{mg} / 100 \mathrm{~g})$ and eggplants $(87 \mathrm{mg} / 100 \mathrm{~g})$. These results are particularly interesting since fruits and vegetables which are generally considered, together with red wines, the best sources of these valuable compounds, had severe limitations. In fact, fruit consumption is limited due to its content of simple carbohydrates, the availability of fresh vegetable and fruits is often limited to the harvest period and the wine consumption should be limited for its content of ethanol and its neurological and carcinogenic effects. On the contrary, the great interest for colored rice is based on their availability all the year round and to the primary position of rice in a balanced human diet, which allows to favorably eat it on an everyday basis. Thus, further studies are in progress aiming and extending the observation to other colored rice cultivars in order to expand their interest as sources of valuable anthocyanins. 
Conflict of interest: The authors declare that they have no conflict of interest. 


\section{REFERENCES}

1. Turrini F, Boggia R, Leardi R, Borriello M, Zunin P (2018) Optimization of the Ultrasonic-Assisted Extraction of Phenolic Compounds from Oryza Sativa L. 'Violet Nori' and Determination of the Antioxidant Properties of its Caryopses and Leaves. Molecules 23:844 Available online at http://www.mdpi.com/1420-3049/23/4/844

2. Zhang M, Zhang RF, Zhang FX, Liu RH (2010) Phenolic profiles and antioxidant activity of black rice bran of different commercially available varieties. J Agric Food Chem 58:7580-7587

3. Bordiga M, Gomez Alonso A, Locatelli M, Travaglia F, Coïsson JD, Hermosin Gutierrez I, Arlorio M (2014) Phenolics characterization and antioxidant activity of six different Oryza sativa cultivars grown in Piedmont (Italy). Food Res Int 65:282-290

4. Goufo P, Trindade H (2014) Rice antioxidants: phenolic acids, flavonoids, anthocyanins, proanthocyanidins, tocopherols, tocotrienols, $\gamma$-oryzanol, and phytic acid. Food Sci Nutr 2:75-104

5. Shao Y, Xu F, Sun X, Bao J, Beta T (2014) Identification and quantification of phenolic acids and anthocyanins as antioxidants in bran, embryo and endosperm of white, red and black rice kernel (Oryza sativa L.). J Cereal Sci 59:211-218

6. Kim MK, Koh HA, Koh K, Kim HS, Lee YS, Kim YH (2008) Identification and quantification of anthocyanin pigments in colored rice. Nutr Res Pract 2:46-49

7. Chiang AN, Wu HL, Yeh HI, Chu CS, Lin HC, Lee WC (2006) Antioxidant effects of black rice extract through the induction of superoxide dismutase and catalase activities. Lipids 41:797-803

8. Nam SH, Choi SP, Kang MY, Koh HJ, Kozukue N, Friedman M (2006) Antioxidative activities of bran from twenty-one pigmented rice cultivars. Food Chem 94:613-620

9. Zhang MW, Zhang RF, Guo BJ, Chi JW, Wei ZC, Xu ZH (2006) The hypolipidemic and antioxidative effects of black rice pericarp anthocyanin in rats. Acta Nutr Sinica 28:404-408

10. Shen, Y, Jin L, Xiao P, Lu Y, Bao JS (2009) Total phenolics, flavonoids, antioxidant capacity in rice grain and their relations to grain color, size and weight. J Cereal Sci 49:106-111

11. Olivas Aguirre FJ, Rodrigo-García J, Martínez-Ruiz ND, Cárdenas-Robles AI, Mendoza-Díaz SO, ÁlvarezParrilla E, González-Aguilar GA, de la Rosa LA, Ramos-Jiménez A, Wall-Medrano A (2016) Cyanidin-3-Oglucoside: physical-chemistry, foodomics and health effects. Molecules 21:1264

12. Vendrame S, Klimis-Zacas D (2018) Anti-inflammatory effect of anthocyanins via modulation of nuclear factor- $\mathrm{\kappa B}$ and mitogen-activated protein kinase signaling cascades. Nutr Rev 73:348-358 
13. Zhao C, Giusti MM., Malik M, Moyer MP, Magnuson BA (2004) Effect of commercial anthocyanin-rich extracts on colonic cancer and nontumorigenic colonic cell growth. J Agric Food Chem 52:2213-2217

14. Tsuda T, Horio F, Osawa T (2002) Cyanidin 3-O-glucoside suppresses nitric oxide production during a zymosan treatment in rats. J Nutr Sci Vitaminol 48:305-310

15. Ponzo V, Goitre I, Fadda M, Gambino R, De Francesco A, Soldati L, Gentile L, Magistroni P, Cassander M, Bo S (2015) Dietary flavonoid intake and cardiovascular risk: A population-based cohort study. J Transl Med $13: 218$

16. Zhu F (2018) Anthocyanins in cereals: Composition and health effects. Food Res Int 109:232-249

17. Marques C, Fernandes I, Meireles M, Faria A, Spencer JPE, Mateus N, Calha C (2018) Gut microbiota modulation accounts for the neuroprotective properties of anthocyanins. Nat Sci Rep 8:11341 Available online at https://www.nature.com/articles/s41598-018-29744-5.

18. Serra D, Almeida LM, Dinis TCP (2018) Dietary polyphenols: A novel strategy to modulate microbiota-gutbrain axis. Trends Food Sci Technol 78:224-233

19. Fang J (2014) Bioavailability of anthocyanins. Drug Metab Rev 46:508--520, DOI: $10.3109 / 03602532.2014 .978080$

20. Yang B, Liua H, Yanga J, Guptac VK, Jianga Y (2018) New insights on bioactivities and biosynthesis of flavonoid glycosides. Trends Food Sci Technol 79:116-124.

21. Fernandes I, De Freitas V, Mateus N (2014) Anthocyanins and human health: How gastric absorption may influence acute human physiology. Nutr Aging 2:1-14

22. Finocchiaro F, Ferrari B, Gianinetti A, Dall'Asta C, Galaverna G, Scazzina F, Pellegrini N (2007) Characterization of antioxidant compounds of red and white rice and changes in total antioxidant capacity during processing. Mol Nutr Food Res 51:1006-1019

23. Hiemori M, Koh E, Mitchell AE (2009) Influence of cooking on anthocyanins in black rice (Oryza sativa L. japonica var. SBR). J Agric Food Chem 57:1908-1914

24. Tang Y, Cai W, Xu B (2016) From rice bag to table: Fate of phenolic chemical compositions and antioxidant activities in waxy and non-waxy black rice during home cooking. Food Chem 191:81-90

25. Singleton VL, Orthofer R, Lamuela-Raventos RM (1999) Analysis of total phenols and other oxidation substrates and antioxidant by means of Folin-Ciocalteu reagent. Meth Enzym 299:152-178

26. Brand-Williams W, Cuvelier ME, Berset C (1995) Use of a free radical method to evaluate antioxidant activity. LWT-Food Sci Technol 28:25-30 
3

4

5

6

7

26.27. Giusti MM, Wrolstad RE (2001) Characterization and measurement of anthocyanins by UV-Visible spectroscopy. In: Current Protocols in Food Analytical Chemistry. Wiley, New York

27.28. Zhaoahua H, Qin P, Zhang Y, Cui S, Ren G (2013) Identification of anthocyanins isolated from black rice (Oriza Sativa L.) and their degradation kinetics. Food Research International 50: 691-697

29. Wu X, Beecher GR, Holden JM, Hayovitz DB, Gebhardt SE, Prior R.L. (2006) Concentrations of Anthocyanins in Common Foods in the United States and Estimation of Normal Consumption. J Agric Food Chem 54:4069-4075X Xu BJ, Chang SKC (2009) Total phenolic, phenolic acid, anthocyanin, flavan-3-ol, and flavonols profiles and antioxidant properties of pinto and black beans (Phaseolus vulgaris L.) as affected by thermal processing. J Agric Food Chem 57:4754-4764

30. Sadilova E, Stintzing F C, Carle R. (2006) Thermal degradation of acylated and nonacylated anthocyanins. J. Food Sci 71:504-512

31. Zaupa M, Calani, L, Del Rio D, Brighenti F, Pellegrini N. (2015) Characterization of total antioxidant capacity and (poly)phenolic compounds of differently pigmented rice varieties and their changes during domestic cooking. Food Chem 187:338-347

32. Yamuangmorn S, Dell B, Prom-U-Thai C (2018) Effects of Cooking on Anthocyanin Concentration and Bioactive Antioxidant Capacity in Glutinous and Non-Glutinous Purple Rice. Rice Science 25: 270-278

33. Wu X, Beecher GR, Holden JM, Hayovitz DB, Gebhardt SE, Prior RL(2006) Concentrations of Anthocyanins in Common Foods in the United States and Estimation of Normal Consumption. J Agric Food Chem 54:4069$\underline{4075}$ 


\section{Figure captions}

Fig. 1. Anthocyanin profile obtained by HPLC. Peak 1, cyanidin-3,5-diglucoside; Peak 2, cyanidin-3-glucoside;

Peak 3, cyanidin-3-rutinoside; Peak 4, peonidin-3-glucoside.

Figure-Fig. 12. The Passing Bablok regression between the total anthocyanins amounts obtained by the UV-VIS (Y axes) and HPLC-DAD (X axes) methods. Results are expressed as $\mu \mathrm{g}$ cyanidin-3-glucoside/mL extracted solution.

Figure-Fig. 23. Total anthocyanins content (mg/100g rice d.m.) in the ten cooked samples (1-10) and in the crude rice (11).

FigureFig. 34. Correlation between the anthocyanins content detected by HPLC-DAD and the RSAs (a, regression line; $b$, residuals) and TPCs (c, regression line; $d$, residuals) of the ten cooked rice samples. Values are expressed as $\mu \mathrm{g} / \mathrm{g}$ dried matter. 
Table 1

The tested cooking conditions

\begin{tabular}{|c|c|c|c|c|c|}
\hline & Appliance & $\begin{array}{l}\text { Cooking } \\
\text { Method }\end{array}$ & $\begin{array}{l}\text { Init. Temp } \\
\quad{ }^{\circ} \mathrm{C}\end{array}$ & $\begin{array}{c}\text { Initial Water } \\
\mathrm{mL}\end{array}$ & $\begin{array}{c}\text { Cooking time } \\
\min \end{array}$ \\
\hline 1 & Stove & Boiling & 20 & 650 & 40 \\
\hline 2 & Stove & Boiling & 100 & 650 & 40 \\
\hline 3 & Stove & Boiling & 20 & 1000 & 40 \\
\hline 4 & Stove & Boiling & 100 & 1000 & 40 \\
\hline 5 & Stove & $\begin{array}{l}\text { Oriental } \\
\text { Cooking }\end{array}$ & 100 & 300 & $\begin{array}{c}14 \text { uncovered }+ \\
14 \text { with a sealed cover }\end{array}$ \\
\hline 6 & Stove & Risotto Cooking & 100 & 1000 & 10 (boiling) + 25 \\
\hline 7 & Stove & $\begin{array}{l}\text { Toasting }+ \\
\text { Risotto Cooking }\end{array}$ & 100 & 750 & $\begin{array}{l}10 \text { (boiling) +3 } \\
\text { (toasting) }+25\end{array}$ \\
\hline 8 & Oven & Pilaf & 20 & 500 & 30 \\
\hline 9 & Oven & Pilaf & 20 & 500 & 3 (toasting) +30 \\
\hline 10 & Oven & Pilaf & 20 & 650 & 10 (boiling) + 10 \\
\hline
\end{tabular}


1

2

3

4

5

6

7

8

9

10

11

12

13

14

15

16

17

18

19

20

21

22

23

24

25

26

27

28

29

30

31

32

33

34

35

36

37

38

39

40

41

42

43

44

45

46

47

48

49

50

51

52

53

54

55

56

57

58

59

60

Figure $x$

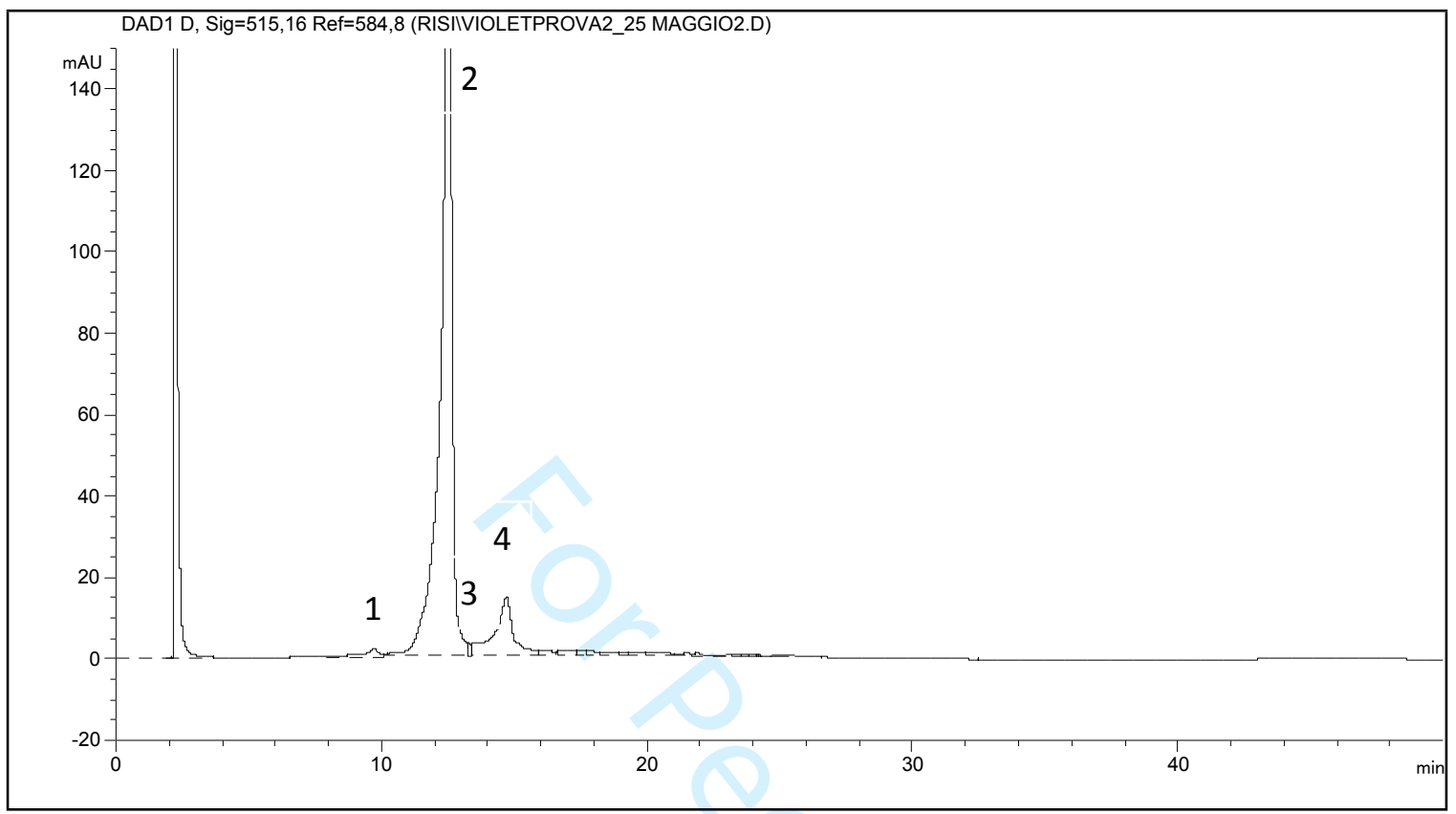

http://mc.manuscriptcentral.com/efrt 
Figure 2

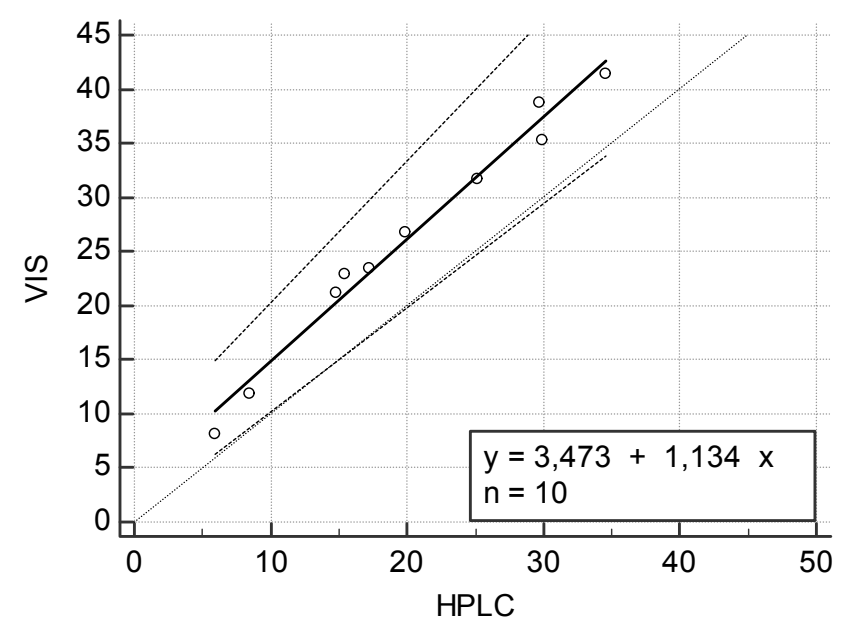


Figure 3

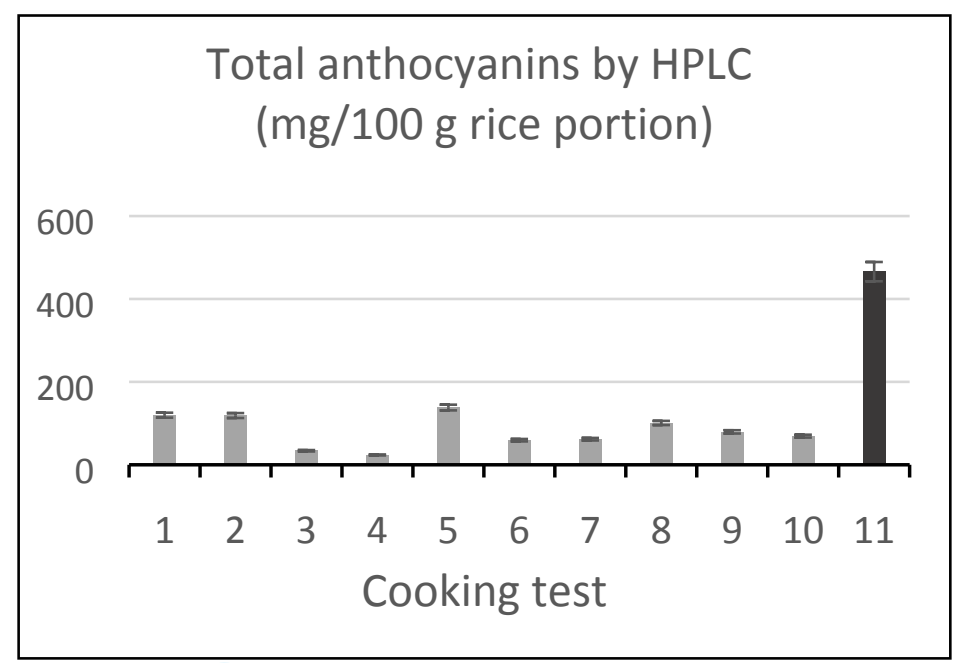

http://mc.manuscriptcentral.com/efrt 


\section{Figure 4}
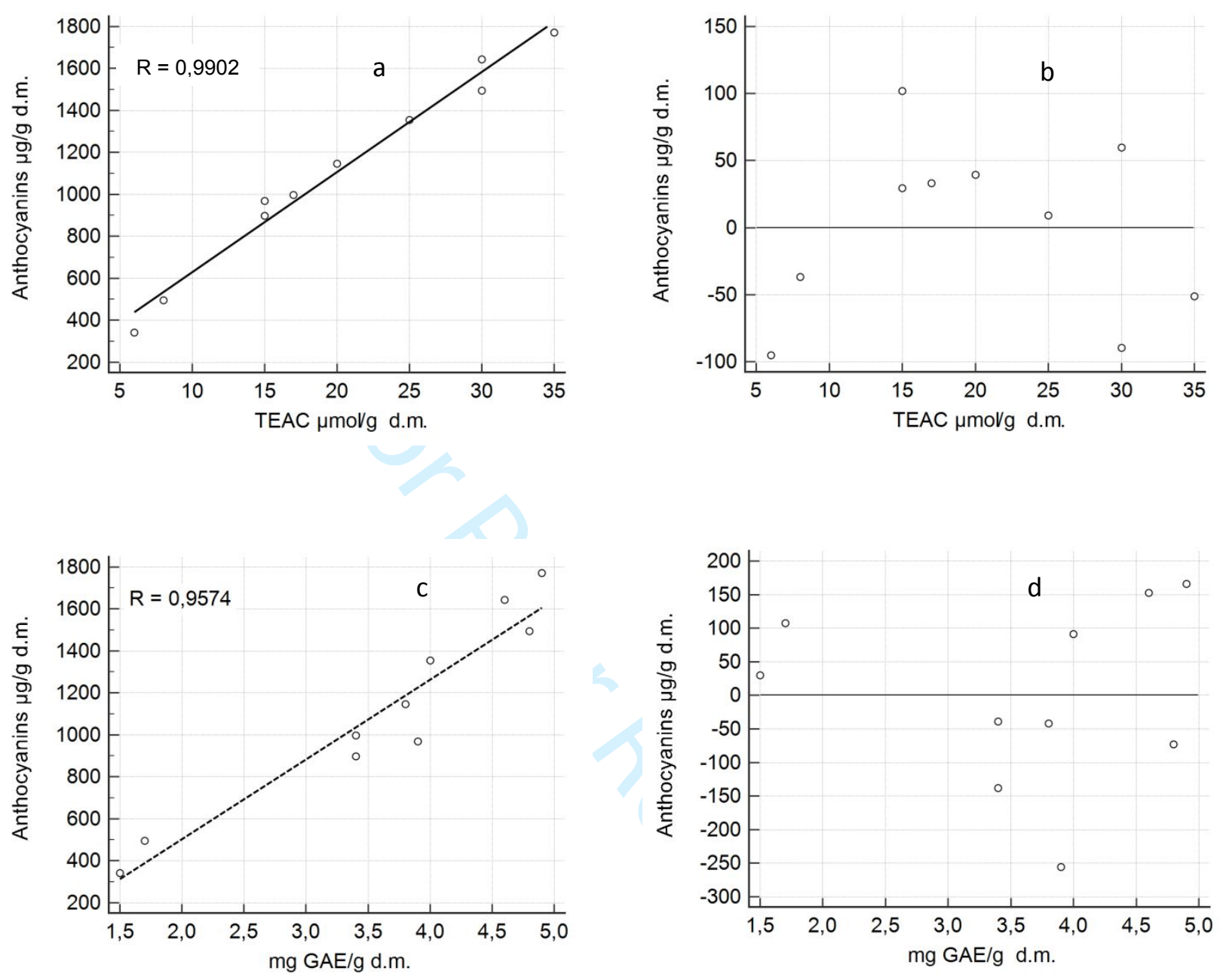
Supplementary materials to

Effects of different cooking conditions on the antioxidant content of a violet rice (Oryza Sativa L. 'Violet Nori')

Silvia Catena, Federica Turrini, Raffaella Boggia, Matilde Borriello, Marco Gardella and Paola Zunin

Corresponding author: Paola Zunin - Department of Pharmacy, University of Genova, Viale Cembrano 4, 16148, Genova, Italy. zunin@difar.unige.it

$\begin{array}{ccccc}\text { Test } & \begin{array}{c}\text { Total Antho by HPLC } \\ (\mu \mathrm{g} / \mathrm{mL} \text { extracted } \\ \text { solution)* }\end{array} & \text { dev.st } & \begin{array}{c}\text { Total Antho by } \mathrm{pH} \\ \text { differential method } \\ (\mu \mathrm{g} / \mathrm{mL} \text { extracted } \\ \text { solution) }\end{array} & \text { dev.st } \\ 1 & 29,9 & 1,7 & 35,3 & 1,9 \\ 2 & 29,7 & 1,4 & 38,8 & 1,8 \\ 3 & 8,5 & 0,5 & 11,9 & 0,6 \\ 4 & 5,9 & 0,3 & 8,1 & 0,3 \\ 5 & 34,5 & 1,3 & 41,4 & 1,4 \\ 6 & 14,8 & 0,7 & 21,2 & 0,8 \\ 7 & 15,4 & 0,6 & 22,9 & 0,7 \\ 8 & 25,2 & 1,5 & 31,8 & 1,6 \\ 9 & 19,8 & 0,9 & 26,8 & 0,8 \\ 10 & 17,3 & 0,9 & 23,4 & 1,1\end{array}$

*expressed as cyanidin-3-glucoside 
Supplementary materials to

Effects of different cooking conditions on the antioxidant content of a violet rice (Oryza Sativa L. 'Violet Nori')

EFRT

Silvia Catena, Federica Turrini, Raffaella Boggia, Matilde Borriello, Marco Gardella and Paola Zunin

Corresponding author: Paola Zunin - Department of Pharmacy, University of Genova,

Viale Cembrano 4, 16148, Genova, Italy. zunin@difar.unige.it

\begin{tabular}{|c|c|c|c|c|c|c|}
\hline Test & $\begin{array}{c}\text { TEAC } \mu \mathrm{mol} / \mathrm{g} \\
\text { (d.m.) }\end{array}$ & dev.st & $\begin{array}{c}\text { TPC } \\
\mathrm{mg} \mathrm{GAE} / \mathrm{g} \text { (d.m.) }\end{array}$ & dev.st & $\begin{array}{l}\text { Anthocyanins } \mu \mathrm{g} / \mathrm{g} \\
\text { (d.m.) * }\end{array}$ & dev.st \\
\hline 1 & 30 & 1,6 & 4,8 & 0,31 & 1495 & 7,0 \\
\hline 2 & 30 & 1,35 & 4,6 & 0,24 & 1644 & 5,8 \\
\hline 3 & 8 & 0,4 & 1,7 & 0,1 & 497 & 2,0 \\
\hline 4 & 6 & 0,36 & 1,5 & 0,09 & 343 & 1,2 \\
\hline 5 & 35 & 1,72 & 4,9 & 0,27 & 1772 & 5,1 \\
\hline 6 & 15 & 0,78 & 3,4 & 1,15 & 898 & 2,8 \\
\hline 7 & 15 & 1 & 3,9 & 0,18 & 970 & 2,5 \\
\hline 8 & 25 & 1,2 & 4,0 & 0,22 & 1355 & 6,0 \\
\hline 9 & 20 & 0,91 & 3,8 & 0,22 & 1146 & 3,8 \\
\hline 10 & 17 & 0,9 & 3,4 & 0,2 & 996 & 3,7 \\
\hline 11 & 67 & 3,57 & 8,8 & 0,43 & 4972 & 30,0 \\
\hline
\end{tabular}

*expressed as cyanidin-3-glucoside 
Supplementary materials to

Effects of different cooking conditions on the antioxidant content of a violet rice (Oryza Sativa L. 'Violet Nori')

EFRT

Silvia Catena, Federica Turrini, Raffaella Boggia, Matilde Borriello, Marco Gardella and Paola Zunin

Corresponding author: Paola Zunin - Department of Pharmacy, University of Genova,

Viale Cembrano 4, 16148, Genova, Italy. zunin@difar.unige.it

$\begin{array}{ccc}\text { Test } & \begin{array}{c}\text { Total antho by HPLC } \\ \mathbf{m g} / \mathbf{1 0 0} \mathbf{~ g}\end{array} & \text { Dev.st } \\ 1 & 119,7 & 7,0 \\ 2 & 118,8 & 5,8 \\ 3 & 33,8 & 2,0 \\ 4 & 23,7 & 1,2 \\ 5 & 138,1 & 5,1 \\ 6 & 59,3 & 2,8 \\ 7 & 61,7 & 2,5 \\ 8 & 100,8 & 6,0 \\ 9 & 79,4 & 3,8 \\ 10 & 69,1 & 3,7 \\ 11 & 465,9 & 30,0 \\ \text { expressed as cyanidine-3-glucoside } & \end{array}$

